\title{
MATRIX SUMMABILITY IN AMENABLE SEMIGROUPS
}

\author{
PETER F. $\mathrm{MAH}^{1}$
}

\begin{abstract}
Necessary and sufficient conditions are given for an infinite matrix to be almost Schur and almost strongly regular in left amenable semigroups.
\end{abstract}

1. Introduction. In [7] the author discusses various methods of matrix summability in amenable semigroups. In that paper, sufficient conditions were given for an infinite matrix to be almost Schur and almost strongly regular. Examples were given to show that the conditions are not necessary. Recently, P. Schaefer gave necessary and sufficient conditions for an infinite matrix to be almost strongly regular for the additive semigroup of positive integers [8]. This was also obtained by Howard T. Bell via a different approach [1]. In this paper, we give necessary and sufficient conditions for an infinite matrix to be almost Schur and almost strongly regular in certain amenable semigroups.

2. Preliminaries. We shall freely use notations and definitions in [7]. Recall that a semigroup $S$ is left amenable (LA) if the Banach space of all bounded real-valued functions on $S$ with the sup norm, $m(S)$, has a normalized positive left translation invariant linear functional. Such a linear functional is called a left invariant mean (LIM). The semigroup is said to be extremely left amenable (ELA) if the LIM is also multiplicative. It is shown by Day [2, p. 524, Theorem 1] that if $S$ is LA, there is a net $\left\{\phi_{x}\right\}$ of finite means converging to left invariance in norm. If, in addition, $S$ is countable then this net can be replaced by a sequence $[6$, p. 42 , Lemma 5.1]. Examples of LA semigroups are commutative semigroups, solvable groups and locally finite groups. Extremely left amenable semigroups are precisely those in which every two elements have a common right zero. For more details, we refer the reader to [2], [3], [4], [5].

Received by the editors October 18,1971 .

AMS (MOS) subject classifications (1969). Primary 4050, 4046, 4031 ; Secondary 4690.

Key words and phrases. Amenable semigroups, extremely amenable semigroups, invariant mean, almost convergence, almost Schur matrices, almost strongly regular matrices.

${ }^{1}$ This research was partially supported by NRC grant A8065. This paper was prepared while the author was a fellow at the 1971 Summer Research Institute of the Canadian Mathematical Congress, University of Alberta, Edmonton, Aiberta.

(c) American Mathematical Society 1973 
If $S$ is LA then a function $f \in m(S)$ is said to be left almost convergent to $k$ if $\phi(f)=k$ for every LIM $\phi$. We shall denote the set of all left almost convergent functions by $F$, and write $f$ lac to $k$ to mean $f$ left almost converges to $k$.

By an infinite matrix $A=(A(s, t))$ on $S$, we shall mean a real-valued function on $S \times S$. If $A$ is an infinite matrix on $S$ and $f \in m(S), A f$ is the function defined on $S$ by $A f(s)=\Sigma_{t} A(s, t) f(t)$, whenever the sum on the right-hand side exists for each $s$ in $S$. (See [7] for the definition of the sum.)

If $\phi$ is a finite mean, i.e., if $\phi$ is a convex combination of point measures in $m(S)^{*}$, define $l_{\phi}: m(S) \rightarrow m(S)$ by

$$
\left(l_{\phi} f\right)(s)=\phi\left(r_{\mathrm{s}} f\right)=\sum_{i=1}^{n_{\phi}} \phi\left(t_{i}\right) f\left(t_{i} s\right),
$$

where $\phi=\sum_{i=1}^{n} \phi\left(t_{i}\right) 1_{\iota_{i}}$. Thus, if $\operatorname{LO}(f)=\left\{l_{s} f: s \in S\right\}$ denotes the left orbit of $f$ and $\operatorname{CoLO}(f)$ denotes the convex hull of the left orbit of $f$, then $l_{\phi} f \in \operatorname{CoLO}(f)$. For the infinite matrix $A=(A(s, t))$, we shall write, for each fixed $t,\left(l_{\phi} A_{t}\right)(s)$ for $\sum_{i=1}^{n_{\phi} \phi} \phi\left(t_{i}\right) A\left(t_{i} s, t\right)$ when we consider $A(s, t)$ as a function of $s$.

We assume throughout that the semigroup $S$ contains no finite left ideals.

3. Almost Schur matrices. An infinite matrix $A$ is said to be almost Schur if $A f \in F$ whenever $f \in m(S)$.

3.1. THEOREM. Let $S$ be a countable $L A$ semigroup. Then the following conditions are both necessary and sufficient for an infinite matrix $A$ to be almost Schur:

(3.1.1) $\operatorname{Sup}_{s} \Sigma_{t}|A(s, t)|<\infty$.

(3.1.2) For each $t, A(s, t)$, as a function of $s$, lac to $\alpha_{t}$.

(3.1.3) For some sequence $\phi_{m}$ of finite means converging to left invariance in norm,

$$
\lim _{m} \sum_{t}\left|\left(l_{\phi_{m}} A_{t}\right)(s)-\alpha_{t}\right|=0 \text { uniformly in } s .
$$

Moreover, Af lac to $\sum_{t} \alpha_{t} f(t)$.

Proof. Assume that $A$ is almost Schur and that, for each $f \in m(S)$, Af lac to $\sum_{t} \alpha_{t} f(t)$. Then (3.1.1) follows from Theorem 3.1 and Lemma 4.1 in [7]. That (3.1.2) holds follows from $A 1_{t}(s)=A 1_{\{t\}}(s)=A(s, t)$, where $l_{B}$ is the characteristic function of $B$. To see (3.1.3), note that for each $f \in m(S)$, we have, by Theorem 7 in [5],

$$
\lim _{m}\left[\left(l_{\phi_{m}} A f\right)(s)-\sum_{t} \alpha_{t} f(t)\right]=0
$$


uniformly in $s$. This is equivalent to

$$
\begin{aligned}
\lim _{m} \sum_{i=1}^{n m} \phi_{m}\left(t_{i}\right) \sum_{t} A\left(t_{i} s,\right. & t) f(t)-\sum_{t} \alpha_{t} f(t) \\
& =\lim _{m} \sum_{t}\left(\sum_{i=1}^{n_{m}} \phi_{m}\left(t_{i}\right) A\left(t_{i} s, t\right)-\alpha_{t}\right) f(t) \\
& =\lim _{m} \sum^{n}\left[\left(l_{\phi_{m}} A_{t}\right)(s)-\alpha_{t}\right] f(t)=0 \text { uniformly in } s .
\end{aligned}
$$

But, for each $s$ and each $m,\left(l_{\phi_{m}} A_{t}\right)(s)-\alpha_{t} \in l_{1}(S)$ and the above set of equations says the sequence $\left(l_{\phi_{m}} A_{t}\right)(s)-\alpha_{t}$ converges weakly to 0 uniformly in $S$ in $l_{1}(S)$. Since weak convergence implies strong convergence for sequences in $l_{1}(S),(3.1 .3)$ follows.

Suppose now that (3.1.1), (3.1.2) and (3.1.3) hold. Using (3.1.1) and (3.1.2) we can show that the sum $\sum_{t}\left|\alpha_{t}\right|$ exists, and thus for every $f \in m(S)$, $\sum_{t} \alpha_{t} f(t)$ exists. Note that, for each $s$ and each $m,\left(l_{\phi_{m}} A_{t}\right)(s)-\alpha_{t} \in l_{1}(S)$, and (3.1.3) implies $\left(l_{\phi_{m}} A_{t}\right)(s)-\alpha_{t}$ converges weakly, i.e., for each $f \in m(S)$,

$$
\begin{aligned}
\lim _{m} \sum_{t}\left[\left(l_{\phi_{m}} A_{t}\right)(s)-\right. & \left.\alpha_{t}\right] f(t) \\
& =\lim _{m} \sum_{i=1}^{n m} \phi_{m}\left(t_{i}\right) \sum_{t} A\left(t_{i} s, t\right) f(t)-\sum_{t} \alpha_{t} f(t) \\
& =\lim _{m}\left[\left(l_{\phi_{m}} A f\right)(s)-\sum_{t} \alpha_{t} f(t)\right]=0 \text { uniformly in } s .
\end{aligned}
$$

By Theorem 7 in [5], we see $A f$ lac to $\sum_{t} \alpha_{t} f(t)$.

3.2. Corollary. A countably infinite $L A$ semigroup $S$ without any finite left ideals cannot have a unique LIM.

Proof. If $S$ has a unique LIM then $F=m(S)$. Thus the identity map $A$, given by $A(s, t)=1$ if $s=t$ and 0 otherwise, is almost Schur. By Theorem 3.1 in [7], $A(s, t)$, as a function of $s$, lac to 0 for each $t$. Hence, for the constant one function 1 on $S, A 1$ lac to 0 by 3.1. But $A 1=1$ and thus must lac to 1 , which cannot be. Thus $S$ cannot have a unique LIM.

3.3. Corollary. A left cancellative LA semigroup without any finite left ideals cannot have a unique LIM.

Proof. This follows immediately from Theorems $E_{1}$ and $E_{2}$ in [6] and 3.2.

3.4. REMARK. The results in 3.2 and 3.3 are already contained in Granirer's works, who actually proved much more than 3.2 and 3.3. We refer the reader to Theorem A, p. 32, and Theorem E, p. 49, in [6]. 
3.5. Example. Let $S$ be the additive positive integers. Define $A$ by

$$
\begin{aligned}
A(m, n) & =1 & & \text { if } m=n, \\
& =0 & & \text { if } n<m, \\
& =-(1 / 2)^{n-m} & & \text { if } n>m .
\end{aligned}
$$

Theorem 3.1 shows $A$ is almost Schur. Condition (3.1.3) is the only difficult part to check. To see this, let $S_{k}=\{i \in S: i \leqq k\}$ and $\phi_{k}=$ $\sum_{i \in S_{k}}(1 / k) 1_{i}$. Then $\phi_{k}$ is a sequence of finite means converging to left invariance in norm. Condition (3.1.3) is equivalent to

$$
\lim _{k} \sum_{n}\left|\sum_{i=1}^{k} \frac{1}{k} A(m+i, n)\right|=0 \quad \text { uniformly in } m .
$$

But

$$
\begin{aligned}
& \lim _{k} \sum_{n}\left|\sum_{i=1}^{k} \frac{1}{k} A(m+i, n)\right| \\
& =\lim _{k} \sum_{n=m+1}^{m+k}\left|\sum_{i=1}^{k} \frac{1}{k} A(m+i, n)\right|+\lim _{k} \sum_{n>m+k}\left|\sum_{i=1}^{k} \frac{1}{k} A(m+i, n)\right| \\
& \leqq \lim _{k}(2 / k)+\lim _{k}(2 / k)=0 \text { uniformly in } m \text {. }
\end{aligned}
$$

4. Almost strongly regular matrices. An infinite matrix $A$ is almost strongly regular if $A f$ lac to $k$ whenever $f$ lac to $k$.

4.1. THEOREM. Let $S$ be a countable LA left cancellative semigroup generated by $B \subset S$. The following conditions are both necessary and sufficient for an infinite matrix $A$ to be almost strongly regular:

(4.1.1) $\operatorname{Sup}_{s} \sum_{t}|A(s, t)|<\infty$.

(4.1.2) $\sum_{t} A(s, t)$, as a function of $s$, lac to 1 .

(4.1.3) For some sequence $\phi_{m}$ of finite means converging to left invariance in norm, $\lim _{m} \sum_{t}\left|\left(l_{\phi_{m}} A_{t}\right)(s)-\left(l_{\phi_{m}} A_{a t}\right)(s)\right|=0$ uniformly in $s$ for every $a \in B$.

Proof. Suppose (4.1.1), (4.1.2) and (4.1.3). Then condition (4.1.1) says $A: m(S) \rightarrow m(S)$ is a bounded linear operator. By (4.1.2) and the fact that $F=C \oplus \mathrm{Cl}(K)$, it suffices to show $A(\mathrm{Cl}(K)) \subset \mathrm{Cl}(K)$, if $C$ and $\mathrm{Cl}(K)$ are as defined on p. 396 in [7]. For each $s \in S, a \in B$ and each $m,\left(l_{\phi_{m}} A_{t}\right)(s)-$ $\left(l_{\phi_{m}} A_{a t}\right)(s) \in l_{1}(S)$; and (4.1.3) says $\left(l_{\phi_{m}} A_{t}\right)(s)-\left(l_{\phi_{m}} A_{a t}\right)(s)$ converges to 0 in $l_{1}$-norm uniformly in $s$. Hence $\left(l_{\phi_{m}} A_{t}\right)(s)-\left(l_{\phi_{m}} A_{a l}\right)(s)$ converges weakly to 0 uniformly in $s$. This implies, for each $f \in m(S)$,

$$
\begin{aligned}
\lim _{m} \sum_{t}\left[\left(l_{\phi_{m}} A_{t}\right)(s)-\left(l_{\phi_{m}} A_{a t}\right)(s)\right] f(a t) & \\
& =\lim _{m}\left[l_{\phi_{m}} A\left(l_{a} f-1_{a S} f\right)\right](s)=0 \text { uniformly in } s .
\end{aligned}
$$


Thus $A\left(l_{a} f-1_{u s} f\right) \in \mathrm{Cl}(K)$. Also, from the following inequality which follows from (5.0.1) in [7],

$$
\begin{aligned}
\mid \sum_{i=1}^{n m} \phi_{m}\left(t_{i}\right) A\left(1_{a S} f\right. & -f)\left(t_{i} s\right) \mid \\
& =\left|-\sum_{i=1}^{n m} \phi_{m}\left(t_{i}\right) \sum_{t \in S \sim a S} A\left(t_{i} s, t\right) f(t)\right| \\
& \leqq\|f\|_{t \in S \sim a S}\left|\left(l_{\phi_{m}} A_{t}\right)(s)\right| \leqq\|f\| \sum_{l}\left|\left(l_{\phi_{m}} A_{t}\right)(s)-\left(l_{\phi_{m}} A_{a t}\right)(s)\right|,
\end{aligned}
$$

we see that $A\left(1_{a S} f-f\right) \in \mathrm{Cl}(K)$. Thus $A\left(l_{n} f-f\right) \in \mathrm{Cl}(K)$. Since

$$
\left\{l_{a} f-f: f \in m(S), a \in B\right\}
$$

spans $K$, and $A$ is continuous, this implies $A(\mathrm{Cl}(K)) \subset \mathrm{Cl}(K)$.

Suppose now that $A$ is almost strongly regular. Then (4.1.1) and (4.1.2) are clear. Hence $A$ is a continuous linear map from $m(S)$ into $m(S)$ such that $A(\mathrm{Cl}(K)) \subset \mathrm{Cl}(K)$.

Now for each $f \in m(S), a \in S$,

$$
A\left(l_{a} f-1_{a S} f\right)(s)=\sum_{t}(A(s, t)-A(s, a t)) f(a t) .
$$

Since $\left(l_{n} f-1_{a S} f\right)=\left(l_{a} f-f\right)+\left(f-1_{a S} f\right)$, by Proposition 4.4 in [7], $l_{a} f-1_{a S} f \in \mathrm{Cl}(K)$. Hence $\sum_{t}(A(s, t)-A(s, a t)) f(a t)$ lac to 0 . By Theorem 7 in [5] it follows that for any sequence $\phi_{m}$ of finite means converging to left invariance in norm,

$$
\begin{aligned}
\lim _{m} l_{\phi_{m}} A\left(l_{a} f-1_{a S} f\right)(s) & \\
& =\lim _{m} \sum_{t}\left[l_{\phi_{m}}\left(A_{\ell}-A_{a^{t}}\right)\right](s) f(a t)=0 \quad \text { uniformly in } s .
\end{aligned}
$$

Since $S$ is left cancellative this implies

$$
\lim _{m} \sum_{t}\left[l_{\phi_{m}}\left(A_{t}-A_{a t}\right)\right](s) g(t)=0 \quad \text { uniformly in } s
$$

for all $g \in m(S)$. But for each $m$ and each $s,\left[l_{\phi_{m}}\left(A_{t}-A_{a t}\right)\right](s)$ is in $l_{1}(S)$; and since weak convergence is equivalent to strong convergence in $l_{1}(S)$ for sequences, it follows that

$$
\lim _{m} \sum_{t}\left|\left(l_{\phi_{m}} A_{t}\right)(s)-\left(l_{\phi_{m}} A_{a t}\right)(s)\right|=0 \quad \text { uniformly in } s .
$$

4.2. Remark. If we take $\phi_{m}$ in (4.1.3) as defined in Example 3.5, then (4.1.3) is just condition (*) on p. 323 in [8].

4.3. THEOREM. Let $S$ be a countable ELA semigroup (but need not be left cancellative). Then the following conditions are both necessary and 
sufficient for an infinite matrix to be almost strongly regular:

(4.3.1) $\operatorname{Sup}_{s} \sum_{t}|A(s, t)|<\infty$.

(4.3.2) $\sum_{t} A(s, t)$, as a function of $s$, lac to 1 .

(4.3.3) For every $a \in S$ such that $a \in S a, \sum_{t \in S \sim a S}|A(s, t)|$, as a function of $s$, lac to 0 .

Proof. That the conditions are sufficient was proved in Theorem 7.3 in [7]. To show that they are necessary we shall only check (4.3.3) since the other two are easy. Let, then, $\left\{t_{m}\right\}$ be a sequence of point measures converging to left invariance in norm. That such a sequence always exists follows from Theorem 3 in [3] and Lemma 5.1 in [6]. Now by Proposition 4.4 in [7], $1_{S \sim a S} f \in \mathrm{Cl}(K)$ for every $f \in m(S)$. Hence, using Theorem 8 in [5],

$$
\begin{aligned}
\lim _{m} A\left(1_{S \sim a S} f\right)\left(t_{m} s\right) & =\lim _{m} \sum_{t} A\left(t_{m} s, t\right) 1_{S \sim a S}(t) f(t) \\
& =\lim _{m} \sum_{t \in S \sim a S} A\left(t_{m} s, t\right) f(t)=0 \quad \text { uniformly in } s .
\end{aligned}
$$

Thus, by defining

$$
\begin{aligned}
B(s, t) & =A(s, t) & & \text { if } t \in S \sim a S, \\
& =0 & & \text { otherwise, }
\end{aligned}
$$

then, for every $f \in m(S)$,

$$
\begin{aligned}
\lim _{m} B f\left(t_{m} s\right) & =\lim _{m} \sum_{t} B\left(t_{m} s, t\right) f(t) \\
& =\lim _{m} \sum_{t \in S \sim_{n} S} A\left(t_{m} s, t\right) f(t)=0 \quad \text { uniformly in } s .
\end{aligned}
$$

But for each $s \in S, B(s, t) \in l_{1}(S)$ and since weak convergence is equivalent to strong convergence in $l_{1}(S)$ for sequences, it follows that

$$
\lim _{m} \sum_{t \in S \sim a S}\left|A\left(t_{m} s, t\right)\right|=\lim _{m} \sum\left|B\left(t_{m} s, t\right)\right|=0 \quad \text { uniformly in } s,
$$

which is precisely condition (4.3.3).

4.4. COROllary. If $A$ is almost strongly regular then $A$ cannot be almost Schur.

Proof. If $A$ is almost strongly regular then $A(s, t)$, as a function of $s$, lac to 0 for each $t$, and $\sum_{t} A(s, t)$, as a function of $s$, lac to 1 . Thus, if $A$ is also almost Schur then $\sum_{t} A(s, t)$, as a function of $s$, lac to 0 .

4.5. Example. Let $S=\{(m, n): m, n$ are positive integers $\}$. Define the binary operation $*$ on $S$ by

$$
\left(m_{1}, n_{1}\right) *\left(m_{2}, n_{2}\right)=\left(m_{1} \vee m_{2}, n_{1} \vee n_{2}\right),
$$


where $m_{1} \vee m_{2}=\max \left\{m_{1}, m_{2}\right\}$. Then it can be checked that $S$ is an ELA semigroup with respect to $*$. Define $A=(A(n, k ; u, v))$ by

$$
\begin{aligned}
A(n, k ; u, v) & =(1 / 2)^{u} & & \text { if } u=v, u \geqq n \vee k, \\
& =0 & & \text { otherwise. }
\end{aligned}
$$

As shown in Theorem 8 in [5] a function $f \in m(S)$, for an ELA $S$, lac to $\alpha$ iff, for every $\varepsilon>0$, there exists a right ideal $S_{\varepsilon}$ in $S$ such that $|f(t)-\alpha|<\varepsilon$ for every $t \in S_{\varepsilon}$. In our semigroup here, this means that for every $\varepsilon>0$, there exist positive integers $n_{0}$ and $k_{0}$ such that for all $n \geqq n_{0}$ and all $k \geqq k_{0},|f(n, k)-\alpha|<\varepsilon$. With this in mind we not show $A$ satisfies the conditions in Theorem 4.2, so that $A$ is almost strongly regular. That $A$ satisfies conditions (4.3.1) and (4.3.2) is easy to check. To see (4.3.3) let $\left(u_{0}, v_{0}\right)$ be given (this is the $a$ in 4.3.3). Then for all $(n, k)$ such that $n>u_{0} \vee v_{0}$ and $k>u_{0} \vee v_{0}$, we have

$$
\sum_{(n, v) \in S \sim\left(u_{0}, v_{0}\right) S}|A(n, k ; u, v)|=\sum_{n<u_{0} \vee v_{0} ; v<u_{0} v_{v_{0}}}|A(n, k ; u, v)|=\sum 0=0 .
$$

Thus, for every $\varepsilon>0$ let $n_{0}>u_{0}$ and $k_{0}>u_{0}$. Then for all $n \geqq n_{0}$ and $k \geqq k_{0}$,

$$
\sum_{(u, r) \in S \sim\left(u_{0}, v_{0}\right) S}|A(n, k ; u, v)|<\varepsilon,
$$

i.e., (4.3.3) holds.

4.6. REMARK. The countability of $S$ was not needed to prove the conditions in the above theorems are sufficient.

\section{BIBLIOGRAPHY}

1. H. T. Bell, A generalization of almost convergence (preprint).

2. M. M. Day, Amenable semigroups, Illinois J. Math. 1 (1957), 509-544. MR 19, 1067.

3. E. E. Granirer, Extremely amenable semigroups, Math. Scand. 17 (1965), 177-197. MR 33 \#5760.

4. - Extremely amenable semigroups. II, Math. Scand. 20 (1967), 93-113. MR $35 \# 3422$.

5. ——, Functional analytic properties of extremely amenable semigroups, Trans. Amer. Math. Soc. 137 (1969), 53-75. MR 39 \#765.

6. - On amenable semigroups with a finite-dimensional set of invariant means. I, II, Illinois J. Math. 7 (1963), 32-58. MR 26 \#1744; \#1745.

7. P. Mah, Summability in amenable semigroups, Trans. Amer. Math. Soc. 156 (1971), 391-403. MR 43 \#771.

8. P. Schaefer, Matrix transformations of almost convergent sequences, Math. $Z$. 112 (1969), 321-325. MR 40 \#7674.

Department of Mathematical Sciences, Lakehead University, Thunder Bay, Ontario, Canada 